\title{
Dynamic Scheduling Model of Bike-Sharing considering Invalid Demand
}

\author{
Liu He $\mathbb{D}^{1,2}$ Tangyi Guo $\mathbb{D}^{1,2}$ and Kun Tang $\mathbb{D}^{1,2}$ \\ ${ }^{1}$ Department of Automation, Nanjing University of Science and Technology, Nanjing, Jiangsu 210094, China \\ ${ }^{2}$ MIIT Key Lab of Traffic Information Fusion \& System Control, Nanjing 210094, China
}

Correspondence should be addressed to Tangyi Guo; transtor@njust.edu.cn

Received 26 June 2020; Revised 19 October 2020; Accepted 2 December 2020; Published 15 December 2020

Academic Editor: Dongfang Ma

Copyright (C) 2020 Liu He et al. This is an open access article distributed under the Creative Commons Attribution License, which permits unrestricted use, distribution, and reproduction in any medium, provided the original work is properly cited.

System resources allocation optimization through dynamic scheduling is key to improving the service level of bike-sharing. This study innovatively introduces three types of invalid demand with negative effect including waiting, transfer, and abandoning, which consists of the total demand of bike-sharing system. Through exploring the dynamic relationship among users' travel demands, the quantity and capacity of bikes at the rental points, the records of bicycles borrowed and returned, and the vehicle scheduling schemes, a demand forecasting model for bike-sharing is established. According to the predicted bikes and the maximum capacity limit at each rental point, an optimization model of scheduling scheme is proposed to reduce the invalid demand and the total scheduling time. A two-layer dynamic coupling model with iterative feedback is obtained by combining the demand prediction model and scheduling optimization model and is then solved by Nicked Pareto Genetic Algorithm (NPGA). The proposed model is applied to a case study and the optimal solution set and corresponding Pareto front are obtained. The invalid demand is greatly reduced from 1094 to 26 by an effective scheduling of 3 rounds and 96 minutes. Empirical results show that the proposed model is able to optimize the resource allocation of bike-sharing, significantly reduce the invalid demand caused by the absence of bikes at the rental point such as waiting in a place, walking to other rental points, and giving up for other travel modes, and effectively improve the system service level.

\section{Introduction}

With the popularization of the concept of sharing economy and green travel, bike-sharing, as an energy-saving, environmental protection, flexible, and healthy way of traveling, is being increasingly popular. Bike-sharing refers to the bikesharing service provided by enterprises in residential areas, commercial areas, public service areas, public transportation stations, campuses, etc. As a new type of sharing economy based on time-sharing rental model, this service has developed rapidly in recent years. According to the Global Development Report on Bike-sharing (2018) [1] published by Cheetah Lab, a global mobile data research firm showed that, in 2019, there were more than 1,000 bike-sharing companies and more than 300 million users. While vigorously developing bike-sharing, various problems have also emerged. The most prominent problem is unavailability of bikes during peak hours which is due to uneven distribution of bikes; some rental points have excessive bikes which sometimes disrupt the road while some rental points have no bikes. In view of this problem, apart from planning the layout and scale of the bike-sharing rental points [2], it is also imperative to establish an accurate and efficient dynamic scheduling system of bike-sharing and maximize the advantages of sharing the economy by improving the service level of system.

The research of bike-sharing scheduling problem mainly focuses on path selection and optimization. Ho and Szeto designed an iterated tabu search heuristic to solve the static bike repositioning problem [3]. Dell'Amico et al. considered the Bike-sharing Rebalancing Problem (BRP) as a special one-commodity pickup-and-delivery capacitated vehicle routing problem and presented four mixed integer linear programming formulations of this problem [4]. Brinkmann 
et al. presented an inventory routing problem for bikesharing systems [5]. Liu et al. proposed a multiobjective model under one hybrid mode with morning fixed and evening demand-responsive by considering the effect on endogenous demands from bike-sharing and studied the influence on feeder transit services [6]. Shi et al. formulated the VRP model for bike-sharing inventory rebalancing and vehicle routing and designed an improved particle swarm optimization (PSO) algorithm to solve this problem [7]. Bulhões et al. established an integer programming model to describe the static bike relocation problem with multiple vehicles and visits [8]. Schuijbroek et al. took service level requirements at each bike-sharing station into account and designed route optimization model. Then, a cluster-first route-second heuristic algorithm was proposed to solve the model [9]. Caggiani et al. proposed a comprehensive dynamic redistribution methodology to mitigate the imbalance of bicycles among zones. The optimal model is aimed to maximize the satisfaction of users and minimize the repositioning cost of vehicles [10]. Guan et al. proposed a layered scheduling strategy for bike-sharing and a static scheduling model with minimum time cost as the objective and vehicle capacity as the constraint is established [11]. Ahmed et al. proposed four upper bounds based on a genetic algorithm, a greedy search algorithm and two hybrid methods that integrate a genetic algorithm, a local search method, and a branch-and-bound algorithm for the balancing problem of bike-sharing stations [12].

In the existing research, dynamic scheduling of bikesharing is defined as vehicle routing problems with time window (VRPTW). Pureza et al. presented a mathematical programming formulation for VRPTW, as well as a tabu search and an ant colony optimization heuristic for obtaining minimum cost routes [13]. Ben Ticha et al. presented an empirical analysis based on VRPTW and solved with branch-and-price algorithms developed for the different types of graphs [14]. Harzi and Krichen proposed a VND approach and benchmark instances were applied to demonstrate the performance [15]. Hernandez et al. proposed the branch-price framework to solve the multitrip vehicle routing problem with time windows [16]. Pierre and Zakaria proposed a stochastic optimized cyclic shift crossover operator for the optimization model with time windows using genetic algorithms [17]. Ma et al. proposed a backpressure-based model with fixed phase sequences for traffic signal optimization under oversaturated networks and a forecasting model for daily traffic flow through a contextual convolutional recurrent neural network as well $[18,19]$.

The above literature mainly focused on the optimization method of path planning algorithm for scheduling vehicles, while most of them considered the demand for bike-sharing trips as static. Actually, as a new mode of transportation, bike-sharing possesses the characteristics of both public transportation and slow traffic.

The problem comes originally from the dynamic contradiction between the existing vehicles, parking space capacity, and the demand for borrowing and returning vehicles. Based on the traffic travel theory, it is the focus of this paper to predict the vehicle demand of each rental point over multiple periods and to formulate the dynamic scheduling scheme. The cause of the problem is the dynamic contradiction between the existing bicycles, capacity, and the demand for borrowing and returning at rental points. Based on the theory of transportation travel, this paper focuses on the multi-period prediction of bicycle demand at each rental point and the formulation of dynamic scheduling scheme.

The rest of the paper is organized as follows: Section 2 presents the procedure of establishing a two-layer dynamic coupling model through 5 steps. Section 3 conducts a case study to illustrate the feasibility and effectiveness. Section 4 concludes with a summary of the main findings in this research and suggestions for future study.

\section{Methodology}

2.1. Problem Description. Bike-sharing is generally concentrated in residential areas, commercial areas, subway stations, and other places with high demand for travel and transfer. Therefore, the continuous distribution of bikesharing in space can be aggregated into a series of discrete rental points on the road network. All bike-sharing users travel from one rental point to another.

The bike-sharing scheduling system consists of a series of bike rental points, scheduling vehicles, and a parking base. The scheduling process is as follows: the command center generates a scheduling scheme according to the scheduling requirements of one or more rental points, which is executed by the scheduling vehicles. The vehicles start from the parking base, load and unload the bikes at multiple rental points according to the scheme, and finally return to the base.

The difference between bike-sharing scheduling problems and general vehicle dynamic scheduling problems is as follows:

(a) Fairness: as a form of public transport, bike-sharing treats all users equally, and there is no indicator of the importance of the rental points.

(b) Flexibility: there is no fixed path or rigid time window for bike-sharing scheduling.

(c) Flow direction: bikes between rental points will generate flow direction according to travel demand; that is, bikes borrowed from one point must return to that point or arrive at another point after a period. This demand is predictable.

(d) Waiting, transfer, and abandoning: when the travel demand at one point is greater than the existing bikes, the excess demand waits in place according to the expected cost, transfers to the nearby rental point (voluntarily looking for bikes), or even cancels (drop for other modes of travel), the probability of which is affected by the user's personal characteristics.

The above characteristics determine that the bikesharing scheduling scheme must be premised on the dynamic change of travel demand. Furthermore, it depends on the relationship between the number of bikes and the demand at the point when the scheduling vehicles arrive. 
Therefore, this paper describes the dynamic scheduling problem of bike-sharing as follows:

The bike-sharing system consists of $H$ scheduling vehicles, 1 parking base, and $N$ rental points.

The scheduling period is from $t_{0}$ to $t_{K}$. The capacity of each rental point is $C_{i}, \quad i=1,2, \cdots, N$. The bikes at rental point $i$ at time $t_{k}, \quad k=0,1, \cdots, K$ is $V_{i}^{t_{k}}$; the total demand of borrowing bikes is indicated as $D_{i}^{t_{k}}$. According to the current status and location of the scheduling vehicle $h$, combined with the borrowing and returning records of the past, the scheduling demand of each rental point is predicted, and a scheduling optimization scheme including scheduling vehicles, scheduling order (set each rental point to be scheduled only once), arrival time, and number of bikes is formulated, so as to ensure the lowest cost of travel for all bike-sharing users at the minimum cost.

2.2. Model Formulation. According to the analysis above, the dynamic scheduling model of bike-sharing includes two submodels: lower demand prediction and upper scheduling optimization. The former is used to establish the dynamic relationship among the users' travel demands, the quantity and capacity of bikes at the rental points, the records of bicycles borrowed and returned, and the vehicle scheduling schemes, while the latter formulates the optimal scheduling scheme according to the prediction results.

2.3. Demand Forecasting Model. Let us start with a definition of the invalid demand of bike-sharing, as shown in Figure 1.

When a user arrives at a rental point, the bike would be successfully borrowed and cycled to another rental point if there are available bikes. Otherwise, the user will face three options: wait in place for other users to return bikes, walk to another rental point to borrow a bike, or give up and switch to other modes of travel.

Let the total demand and returned bikes at the time of $t_{k}$ rental point $i$ be $L_{i}^{t_{k}}$ and $R_{i}^{t_{k}}$, respectively, and the scheduling amount is $S_{i}^{t_{k}}$; then, the bikes at the next time $V_{i}^{t_{k+1}}$ are the result of the current bikes $V_{i}^{t_{k}}$ minus the total demand bikes $L_{i}^{t_{k}}$, plus the valid returned bikes $R_{i}^{t_{k}}$ as well as the scheduling bikes $S_{i}^{t_{k}}$ :

$$
V_{i}^{t_{k+1}}=V_{i}^{t_{k}}-L_{i}^{t_{k}}+R_{i}^{t_{k}}+S_{i}^{t_{k}} .
$$

The components are shown as follows.

2.3.1. Valid Demand $L_{i}^{t_{k}}$. If the current bikes meet the total demand for borrowing at this time, then the valid demand is the total demand; otherwise, all bikes will be borrowed. The valid demand is represented as

$$
L_{i}^{t_{k}}= \begin{cases}D_{i}^{t_{k}}, & D_{i}^{t_{k}} \leq V_{i}^{t_{k}}, \\ V_{i}^{t_{k}}, & D_{i}^{t_{k}}>V_{i}^{t_{k}} .\end{cases}
$$

2.3.2. Invalid Demand $\widetilde{L}_{i}^{t_{k}}$. The invalid demand $\widetilde{L}_{i}^{t_{k}}$ can be expressed as

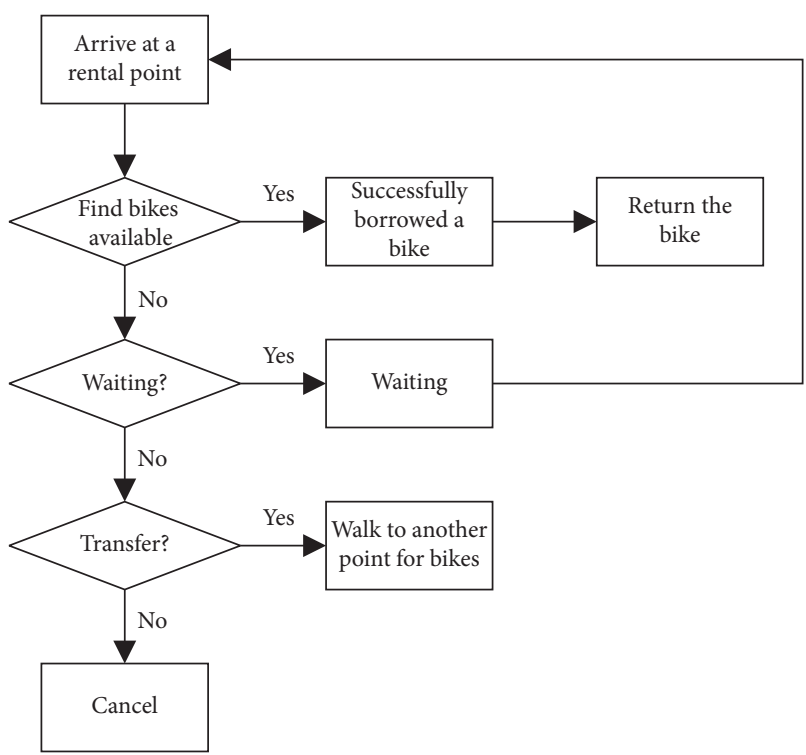

FIgURE 1: The invalid demand of bike-sharing.

$$
\widetilde{L}_{i}^{t_{k}}=D_{i}^{t_{k}}-V_{i}^{t_{k}}=W_{i}^{t_{k}}+T_{i}^{t_{k}}+A_{i}^{t_{k}}, \quad D_{i}^{t_{k}}>V_{i}^{t_{k}} .
$$

In the formula, $\widetilde{L}_{i}^{t_{k}}$ represents the invalid number of bikes borrowed by time $t_{k}$ and at rental point $i . W_{i}^{t_{k}}, T_{i}^{t_{k}}$, and $A_{i}^{t_{k}}$ represent the number of waiting, transfer, and abandoning of bikes by time $t_{k}$ at the rental point $i$.

The following assumptions are made for the invalid demand and formulas (4) to (7) are obtained:

(a) The waiting probability $p w_{i}^{t_{k}}$ of the rental point $i$ is positively correlated with the valid returned bikes during this period and negatively correlated with the waiting time and the users waiting in the previous period.

(b) The transfer probability $p t_{i j}^{t_{k}}$ of walking from the rental point $i$ to rental point $j$ is positively correlated with the waiting users at the last time period of the rental point $i$, and the number of bikes that can be borrowed at rental point $j$, and negatively correlated with the expected returned bikes, and shortest path distance between rental points $i$ and $j$.

(c) The abandoning probability $p a_{i}^{t_{k}}$ at rental point $i$ is positively correlated with the waiting users in the previous period and negatively correlated with the expected returned of bikes at point $i$ during this period.

(d) All invalid users only make one choice within one period and cannot be changed.

$$
\begin{gathered}
p w_{i}^{t_{k}}=\theta_{1} \cdot p w_{i}^{t_{k-1}} \cdot \frac{R_{i}^{t_{k}}}{W_{i}^{t_{k-1}} C_{i}}, \\
p t_{i j}^{t_{k}}=\theta_{2} \cdot \frac{W_{i}^{t_{k-1}} \cdot V_{j}^{t_{k}}}{R_{i}^{t_{k}} \cdot d_{i j}},
\end{gathered}
$$




$$
\begin{aligned}
p a_{i}^{t_{k}} & =\theta_{3} \cdot \frac{W_{i}^{t_{k-1}}}{R_{i}^{t_{k}}}, \\
p w_{i}^{t_{k}}+\sum_{j=1}^{N} p t_{i j}^{t_{k}}+p a_{i}^{t_{k}} & =1 .
\end{aligned}
$$

In the formula, $\theta_{1}, \theta_{2}, \theta_{3}$, and $p w_{i}^{t_{0}}$ are all undetermined coefficients greater than 0 , which are related to the user's individual characteristics, and can be determined by SP/RP survey. $d_{i j}$ in formula (5) represents the shortest path distance between the rental points $i$ and $j$, which is generally implemented by Dijkstra algorithms. Equation (7) guarantees that the sum of the probabilities of waiting, transfer, and abandoning is 1 .

The above formula is summed up to get the amount of waiting, transfer, and abandoning in the invalid demand of time $t_{k}$ at rental point $i$.

$$
\begin{aligned}
T_{i}^{t_{k}} & =\sum_{s=1}^{\widetilde{L}_{i}^{k_{i}}} p t_{i}^{t_{k}}, \\
& =\theta_{2} \frac{V_{j}^{t_{k}}}{R_{i}^{t_{k}} \cdot r_{i j}} \sum_{s=1}^{\widetilde{L}_{i}^{k_{k}}} W_{i}^{t_{k-1}}, \\
W_{i}^{t_{k}} & =\sum_{s=1}^{\widetilde{L}_{i}^{t_{k}}} p w_{i}^{t_{k}}, \\
& =\theta_{1} \frac{R_{i}^{t_{k}}}{C_{i}} \sum_{s=1}^{\widetilde{L}_{i}} \frac{p w_{i}^{t_{k-1}}}{W_{i}^{t_{k-1}}}, \\
A_{i}^{t_{k}} & =\sum_{s=1}^{\widetilde{L}_{i}^{k}} p a_{i}^{t_{k}}, \\
& =\theta_{3} \frac{1}{R_{i}^{t_{k}}} \sum_{s=1}^{\widetilde{L}_{i}^{t_{k}}} W_{i}^{t_{k-1}} .
\end{aligned}
$$

2.3.3. Valid Return $R_{j}^{t_{k}}$. The valid return bikes are determined by the valid demand at the starting rental point and the distribution probability matrix between the starting point and the ending point. The latter is defined as

$$
\begin{aligned}
P^{t_{k}} & =\left(\begin{array}{ccc}
P_{11}^{t_{k}} & \cdots & P_{1 N}^{t_{k}} \\
\vdots & P_{i j}^{t_{k}} & \vdots \\
P_{N 1}^{t_{k}} & \cdots & P_{N N}^{t_{k}}
\end{array}\right), \\
\text { s.t. } 0 & \leq P_{k j} \leq 1, \\
\sum_{j=1}^{n} P_{k j} & =1, \quad k=1,2, \ldots, n .
\end{aligned}
$$

In the formula, $P_{i j}^{t_{k}}$ represents the probability of borrowing bikes from rental point $i$ at time $t_{k}$ and returning them at rental point $j$, which can be obtained from the operation records of bike-sharing.
Since bikes are generally ridden in nonmotorized lanes, the capacity limit of the road is generally not considered, so the shortest path of distance can be used for conversion. The valid return $R_{j}^{t_{k}}$ at rental point $j$ is expressed as

$$
R_{j}^{t_{k}}=\sum_{i=1}^{N} L_{i}^{t_{k}-\left(d_{i j} / v\right)} P_{i j}^{t_{k}-\left(d_{i j} / v\right)}
$$

where $v$ represents the average speed of the bike.

2.3.4. Scheduling $S_{i}^{t_{k}}$. Set each rental point to be scheduled only once, so $S_{i}^{t_{k}}$ is abbreviated as $S_{i}$. The scheduling bikes at rental point $i$ are determined by scheduling optimization model. $S_{i}<0$ indicates loading at the rental point, while $S_{i}>0$ indicates unloading.

2.4. Scheduling Optimization Model. The optimization model for scheduling is based on the predicted result of bikes and the maximum capacity at each rental point. On the one hand, the optimal scheduling scheme is to ensure the service level and reduce the invalid demand. On the other hand, it will also reduce the scheduling cost and improve the efficiency of the operating enterprise. This is a multiobjective problem and the objective function is established as follows:

$$
\begin{aligned}
& \min \left(Z_{1}\right)=\sum_{k=0}^{K} \sum_{i=1}^{N}\left(\beta_{1} W_{i}^{t_{k}}+\beta_{2} T_{i}^{t_{k}}+\beta_{3} A_{i}^{t_{k}}\right), \\
& =\sum_{k=1}^{K} \sum_{i=1}^{N}\left(\beta_{1} \theta_{1} \frac{R_{i}^{t_{k}}}{C_{i}} \sum_{s=1}^{\widetilde{L}_{i}^{t}} \frac{p w_{i}^{t_{k-1}}}{W_{i}^{t_{k-1}}}+\beta_{2} \theta_{2} \frac{V_{j}^{t_{k}}}{R_{i}^{t_{k}} \cdot r_{i j}} \sum_{s=1}^{\widetilde{L}_{i}^{t_{k}}} W_{i}^{t_{k-1}}\right. \\
& \left.+\beta_{3} \theta_{3} \frac{1}{R_{i}^{t_{k}}} \sum_{s=1}^{\widetilde{L}_{i}^{k}} W_{i}^{t_{k-1}}\right) \\
& \text { s.t. } \forall V_{i}^{t_{k}}=0 \bigcup V_{i}^{t_{k}}>C_{i} B_{j}^{t_{i \times d t}}<0, B_{l}^{t_{i \times d t}}>C_{l} \text {, } \\
& \min \left(Z_{2}\right)=\sum_{h=1}^{H} \sum_{i=0}^{N} \sum_{j=0, j \neq i}^{N}\left(\text { QUOTE } t_{a} t_{a} X_{i j h}+\gamma_{0}\left|S_{i}\right|\right), \\
& \text { s.t. } i=0, \sum_{h=1}^{H} \sum_{j=0}^{N} X_{i j h}=H, \sum_{h=1}^{H} y_{h i}=1,\left|\sum_{i=0}^{N} \sum_{j=0, j \neq i}^{N} S_{i} X_{i j h}\right| \leq Q_{h} \text {, } \\
& \forall h=1,2, \cdots, H, t_{j} \\
& =\sum_{h=1}^{H} \sum_{j=0, j \neq i}^{N} X_{i j h}\left(t_{i}+\gamma_{i j}+\gamma_{0}\left|S_{i}\right|\right), X_{i j h} \\
& =\left\{\begin{array}{l}
1, \text { vehicle } h \text { drive from rental point } i \text { to } j, \\
0, \text { otherwise, }
\end{array} y_{h i}\right. \\
& =\left\{\begin{array}{l}
1, \text { rental point } i \text { is served by vehicle } h \\
0, \text { otherwise }
\end{array}\right.
\end{aligned}
$$


In the formula, $t_{a}$ represents the travel time of the scheduling vehicle from rental point $i$ to $j, \gamma_{0}$ represents the average loading and unloading time of one bike, $H$ is the total scheduling vehicles, and $Q_{h}$ is the maximum load of vehicle $h$.

The objective function (14) indicates that the minimum invalid demand has a negative effect on the service level of bike-sharing. Since this is a Soft Time Windows Problem, the time window constraints are incorporated into the target function by introducing waiting penalty factor $\beta_{1}$, transfer penalty factor $\beta_{2}$, and abandoning penalty factor $\beta_{3}$. Condition (15) indicates that the schedule needs to meet the situation where there is no bike to borrow or the rental point exceeds its capacity.

The objective function (16) indicates that the scheduling cost of an enterprise is minimized, which is represented by the sum of the driving time, the loading, and unloading time of the bikes. Condition (17) indicates that there are $H$ paths for departure from the rental point and $H$ paths for arrival. Condition (18) indicates that the rental point $i$ can only schedule one bike, condition (19) indicates that the loading number of each vehicle does not exceed the capacity, condition (20) indicates that the time to reach the rental point $j$ is equal to the time when the vehicle reaches point $i$ plus the loading and unloading time at point $j$ and the travel time from point $i$ to point $j$.

2.5. Model Solution. The lower demand prediction model regards all the bike-sharing rental points in the area as a whole system, and it is appropriate to establish a system dynamics simulation model to solve the changes of system characteristics in the subsequent moments with the current user demand, historical travel distribution, and scheduling scheme for the input and deduction. The upper level scheduling optimization model is a multiobjective optimization problem with a time window (vehicle routing problems with time windows, VRPTW) that determines the time and order of the scheduling vehicle through each rental point based on the dual objectives of invalid demand and enterprise scheduling cost. It is generally solved by heuristic algorithm [20].

In the simulation process, the predicted scheduling demand is influenced by the number of past bikes borrowed, which is the basis for the development of scheduling scheme, which will affect the demand prediction results of all rental points in the process of development. At the same time, the implementation of scheduling affects the valid demand and pushes the time forward. Compare the difference between valid and predicted demand to determine whether the scheduling scheme needs to be adjusted. When the actual time reaches the end, the result will be output. The simulation process is shown in Figure 2.

Throughout the simulation process, on the one hand, demand prediction and scheduling optimization are intertwined with constant feedback; on the other hand, scheduling optimization is a multiobjective VRP problem, which needs to be considered separately. For the traditional VRP problem, heuristic algorithm is generally used to solve the problem. The selection of bike-sharing requirement path needs the support of modelled and quantitative methods, and it has the complete nature of NP. Genetic algorithm has some outstanding advantages for solving this kind of problem. Since the submodel of bike-sharing scheduling involves two objective equations (11), (12) of user service level and enterprise scheduling cost, it is advisable to adopt a multiobjective genetic algorithm (MOGA). This method is an algorithm developed in recent years to deal with multiobjective optimization problems using genetic algorithms. Traditional methods are difficult to deal with large-scale problems; MOGA not only can deal with large-scale problems but also is not limited by the nature of the problem (linear, continuous, microusability, multipeak, etc.) and can search for the global optimal solution of the problem. It is also independent of the form of Pareto's optimal frontier compared to conventional optimization methods [15]. We choose the Ned Pareto Genetic Algorithm (NPGA) which is more economical to calculate.

The specific model solving steps are as follows.

Step 1. Starting from $t_{0}$, the matrix is constructed by the demand forecasting model to predict the number of bikes at each rental point at any time in the future based on historical bikes borrowing records.

$$
V=\left(\begin{array}{ccc}
V_{1}^{t_{0}} & \cdots & V_{N}^{t_{0}} \\
\vdots & V_{i}^{t_{k}} & \vdots \\
V_{1}^{t_{K}} & \cdots & V_{N}^{t_{K}}
\end{array}\right) .
$$

Step 2. Select $n(n \leq N)$ rental points to be scheduled from $V$ to satisfy $V_{i}^{t_{k}}=0$ or $V_{i}^{t_{k}}>C_{i}$ or other safety inventory conditions. If there is no rental point that needs to be scheduled, advance a time step and then move on to step 1; otherwise, move to step 3 .

Step 3. Develop a scheduling scheme, when a rental point is determined; $V$ needs to be updated. The scheme includes the scheduling sequence and the scheduling number of each rental point $S_{i}$.

Step 3.1. The construction of the solution and the generation of the initial group.

A string of genes is used to represent a group of bikesharing rental point scheduling schemes. Each gene is a positive integer variable of length $N$, where each represents the order in which the corresponding alternative locations are accessed, such as $\{3,6,1,2,5$, $4\}$. According to the structure of the solution, $L$ individuals are randomly generated to form the initial group, which is denoted as $G_{0}=\left\{g_{1}, g_{2}, \cdots, g_{L}\right\}$.

Step 3.2. Adaptive Equation

The adaptive equation reflects the function of the individual's proximity to the optimal solution in the optimization calculation, and the adaptability equation for multiobjective problems can be directly objective equation (11) and (13).

Step 3.3. Selection

The selection of Pareto genetic algorithm is based on league selection of league-size 2, using a dynamically 


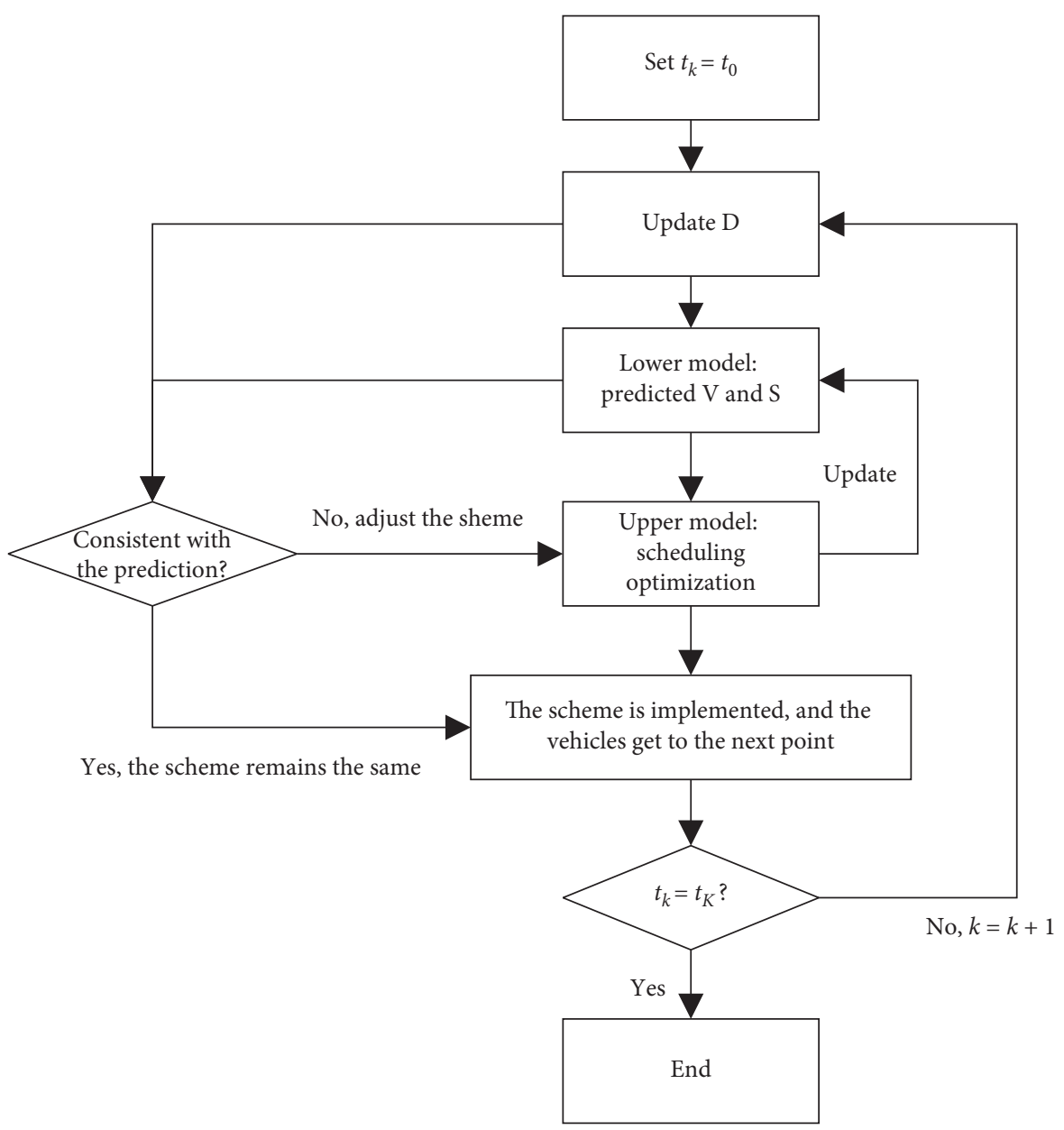

FIgURE 2: Simulation flow chart of bike-sharing dynamic scheduling system.

updated niche strategy. In league selection, two individuals are randomly selected from a parental population. Compare them with subpopulations originally selected from the population. The two individuals are superior to everyone in the subpopulation. If one of the individuals is not inferior to all individuals of this subpopulation and the other is at least inferior to one individual of this subpopulation, then select the former, and if all of them are inferior to at least one individual of this subpopulation or none of them are inferior to any individual of this subpopulation, then examine the two individuals in the current subpopulation. This method is done by placing the two individuals in separate subpopulations and calculating their totals; the individual with a smaller total number wins the game.

Step 3.4. Crossover

Since the structure of the solution is nonbinary, the method of selecting the crossing point and simply cross recombination cannot be possible. The specific method is to select the father individual and the mother individual from the population and randomly determine the crossing of the two parents, such as $\{3$, $6,1,2,5,4\}$ and $\{1,2|3,4| 5,6\}$, the cross-produced child is the part between the cross points of the mother, followed by the parent, and the repeated numbers in the child are removed, that is, $\{3,4,6,1,2$, $5\}$.

Step 3.5. Mutations

The specific operation of the mutation is to select two of the mutated individuals and exchange their positions. For example, 1 and 2 of $\{3,6,1,2,5,4\}$ are mutated to $\{6,3,1,2,5,4\}$.

\section{Step 4. Scheduling Implementation}

When a new rental point had been reached and the current time is recorded after loading and unloading, the scheduling is ended when $t=t_{K}$; otherwise, go to step 5 .

Step 5. Check whether the valid bikes at each rental point are consistent with the predicted value, and if consistent, delete the current point from the scheduling scheme and go to step 4; otherwise, return to step 1 .

\section{Case Study}

A bike-sharing travel scenario in the peak hour is built to test the model. Assume that there are 8 bike-sharing rental points and 1 parking base in this area as shown in Figure 3, 


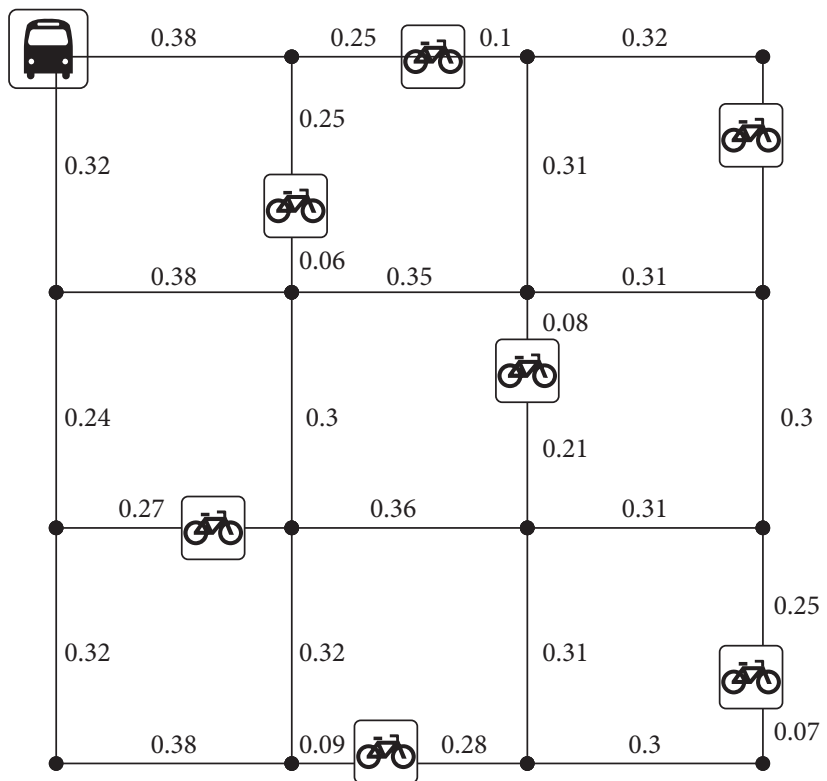

FIGURE 3: Location of bike-sharing rental points and parking lot.

and the numbers in this figure mean the length of each link and the unit is kilometers. The simulation starts at 7:00 am and ends at 9:00 am with a time interval of 5 minutes. The capacity of each rental point, the number of initial bikes, and the demand by every time interval are shown in Table 1, and the probability distribution of bike-sharing travel destinations is shown in Table 2. The capacity of the parking base is infinity. The rental point will be added to the scheduling scheme if the bikes are all gone or exceed the capacity when the vehicle arrives.

One scheduling vehicle departs from the parked base and eventually returns. The maximum loading is 300 , and each rental point is scheduled only once. Regardless of the influence of road traffic, the speed of walking, bike-sharing, and scheduling vehicle is $5 \mathrm{~km} / \mathrm{h}, 8 \mathrm{~km} / \mathrm{h}$, and $30 \mathrm{~km} / \mathrm{h}$, respectively, and the average loading and unloading time is 3 seconds per bike. The bikes for each period without scheduling are shown in Table 3.

The bikes at points 1 and 5 exceed the capacity, and at points 3, 4, 7, and 8 the bikes are empty. Only points 2 and 6 do not need to be scheduled.

The multiobjective optimization model with the lowest cost and minimum invalid demand was established by using the Niche Pareto genetic algorithm. Suppose that the population size is 50, the maximum evolutionary algebra is 100 , the probability of crossover is 0.9 , the probability of mutation is 0.04 , the waiting penalty factor $\beta_{1}$ is 1 , the transfer penalty factor $\beta_{2}$ is 2 , and the abandoning penalty factor $\beta_{3}$ is 3 . Taking the product of the objective functions (19) and (21) as the optimal solution, after multiple rounds of iteration (the evolution process is shown in Figure 2), the optimal scheduling scheme is 7-46-8-3-1-5-2.

As shown in Figure 4, the overall evolution obtained the optimal solution for the first time in the 20th generation and remained stable in the 49 th generation.
TABLE 1: Capacity, initial number, and demand of bike-sharing.

\begin{tabular}{lccc}
\hline Point & $C_{i}$ & $V_{i}^{t_{0}}$ & $D_{i}^{t_{k}}$ (random) \\
\hline 1 & 100 & 45 & $1-5$ \\
2 & 100 & 50 & $1-5$ \\
3 & 120 & 65 & $6-10$ \\
4 & 110 & 50 & $6-10$ \\
5 & 120 & 55 & $1-5$ \\
6 & 80 & 40 & $1-5$ \\
7 & 90 & 45 & $6-10$ \\
8 & 70 & 65 & $6-10$ \\
\hline
\end{tabular}

TABle 2: Travel destination selection probability of bike-sharing.

\begin{tabular}{lccccccccc}
\hline Point & 1 & 2 & 3 & 4 & 5 & 6 & 7 & 8 & Sum \\
\hline 1 & 0 & 0 & 0 & 0 & 0.2 & 0.2 & 0.3 & 0.3 & 1 \\
2 & 0 & 0 & 0 & 0 & 0.5 & 0.1 & 0.2 & 0.2 & 1 \\
3 & 0 & 0 & 0 & 0 & 0.1 & 0.5 & 0.3 & 0.1 & 1 \\
4 & 0 & 0 & 0 & 0 & 0.2 & 0.2 & 0.1 & 0.5 & 1 \\
5 & 0.2 & 0.5 & 0.1 & 0.2 & 0 & 0 & 0 & 0 & 1 \\
6 & 0.3 & 0.1 & 0.4 & 0.2 & 0 & 0 & 0 & 0 & 1 \\
7 & 0.1 & 0.1 & 0.3 & 0.5 & 0 & 0 & 0 & 0 & 1 \\
8 & 0.2 & 0.4 & 0.2 & 0.2 & 0 & 0 & 0 & 0 & 1 \\
\hline
\end{tabular}

Figure 5 reflects the Pareto frontier corresponding to the optimal solution set of the double objective function, and the weights can be further clarified according to valid demand.

The bikes at each rental point under the optimal scheme are shown in Table 4, and the records of vehicle are shown in Table 5.

It can be seen from Table 3 to Table 5 that, after the scheme is adopted, the vehicle unloads bikes at points $3,4,7$, and 8 and loads bikes at points 1 and 5. Most of the time, there will be a bike to borrow and a place to return. Through three rounds of effective scheduling which takes 96 minutes, the invalid demand is greatly reduced from 1094 to 26, and the bike-sharing resource allocation is better. 
TABle 3: Numbers of bikes for each period without scheduling.

\begin{tabular}{lcccccccccccccccccccccccccc}
\hline Point & 1 & 2 & 3 & 4 & 5 & 6 & 7 & 8 & 9 & 10 & 11 & 12 & 13 & 14 & 15 & 16 & 17 & 18 & 19 & 20 & 21 & 22 & 23 & 24 \\
\hline 1 & 41 & 40 & 43 & 51 & 61 & 69 & 76 & 86 & 91 & 100 & 103 & 110 & 111 & 115 & 113 & 111 & 112 & 114 & 117 & 117 & 117 & 117 & 116 & 120 \\
2 & 47 & 45 & 46 & 47 & 48 & 48 & 48 & 49 & 49 & 51 & 53 & 54 & 55 & 55 & 56 & 52 & 49 & 49 & 49 & 48 & 45 & 42 & 41 & 41 \\
3 & 56 & 48 & 43 & 40 & 36 & 35 & 33 & 32 & 32 & 30 & 23 & 21 & 19 & 13 & 5 & 0 & 0 & 0 & 0 & 0 & 0 & 0 & 0 & 0 \\
4 & 44 & 36 & 33 & 27 & 25 & 23 & 20 & 14 & 8 & 3 & 0 & 0 & 0 & 0 & 0 & 0 & 0 & 0 & 0 & 0 & 0 & 0 & 0 & 0 \\
5 & 52 & 56 & 63 & 71 & 79 & 89 & 96 & 106 & 113 & 122 & 127 & 139 & 143 & 150 & 156 & 162 & 165 & 168 & 172 & 170 & 175 & 180 & 182 & 184 \\
6 & 38 & 38 & 40 & 40 & 38 & 41 & 40 & 41 & 44 & 45 & 47 & 47 & 48 & 49 & 47 & 48 & 47 & 49 & 45 & 45 & 46 & 46 & 45 & 42 \\
7 & 38 & 31 & 29 & 25 & 19 & 14 & 6 & 0 & 0 & 0 & 0 & 0 & 0 & 0 & 0 & 0 & 0 & 0 & 0 & 0 & 0 & 0 & 0 & 0 \\
8 & 55 & 47 & 40 & 35 & 27 & 22 & 15 & 9 & 4 & 0 & 0 & 0 & 0 & 0 & 0 & 0 & 0 & 0 & 0 & 0 & 0 & 0 & 0 & 0 \\
\hline
\end{tabular}

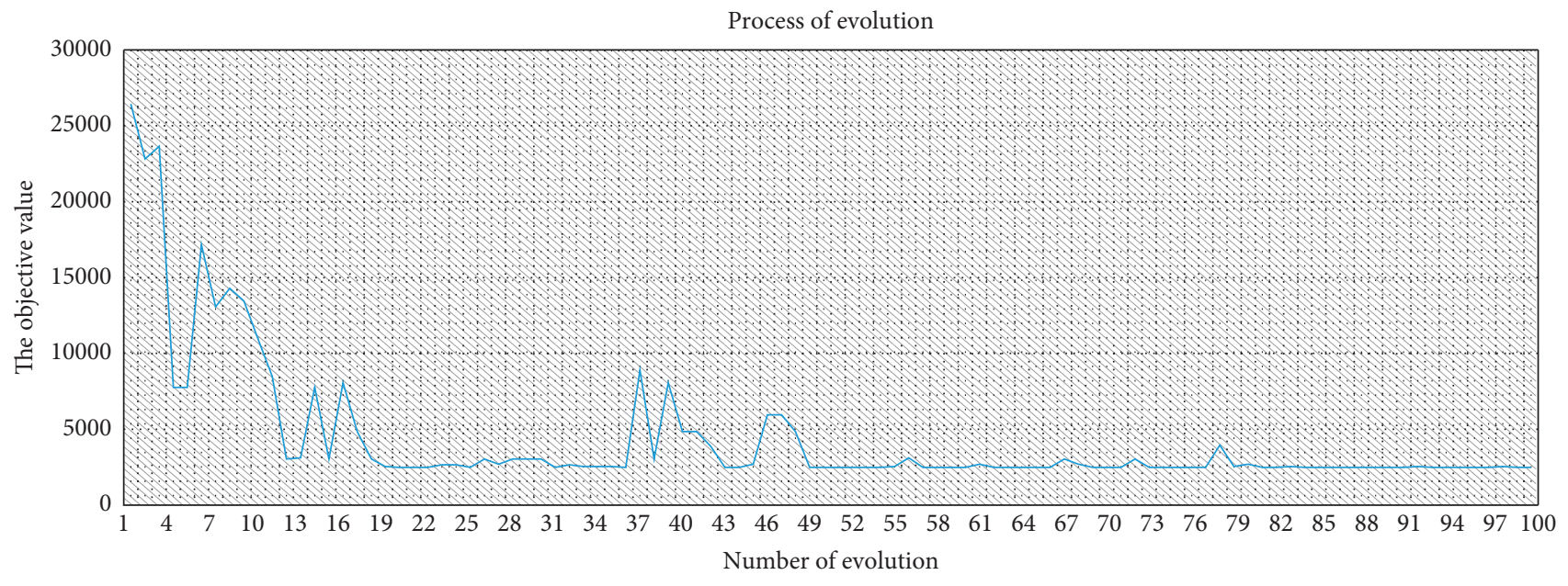

Figure 4: Process of evolution.

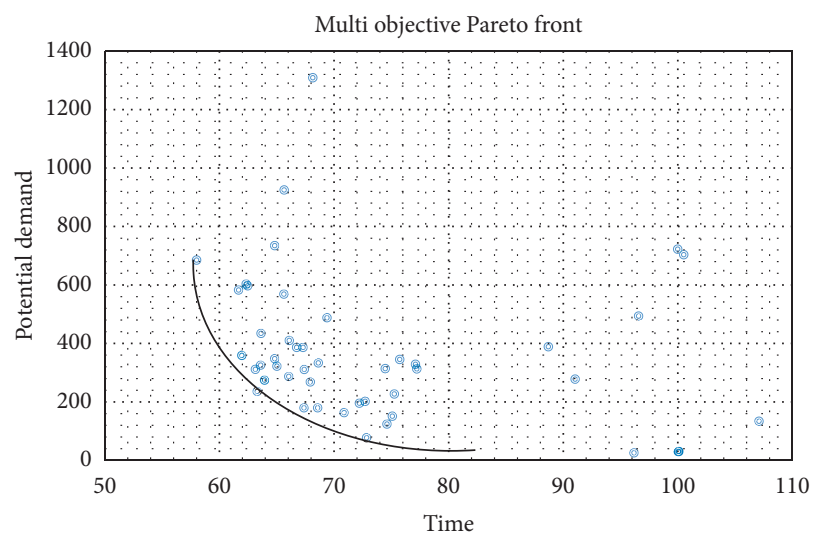

Figure 5: Pareto frontier.

TABLE 4: Numbers of bikes for each period in the optimal scheduling scheme.

\begin{tabular}{lcccccccccccccccccccccccc}
\hline Point & 1 & 2 & 3 & 4 & 5 & 6 & 7 & 8 & 9 & 10 & 11 & 12 & 13 & 14 & 15 & 16 & 17 & 18 & 19 & 20 & 21 & 22 & 23 & 24 \\
\hline 1 & 41 & 40 & 43 & 51 & 61 & 69 & 76 & 86 & 91 & 101 & 106 & 116 & 121 & 9 & 12 & 18 & 24 & 32 & 40 & 44 & 48 & 54 & 58 & 67 \\
2 & 47 & 45 & 46 & 47 & 48 & 48 & 48 & 49 & 49 & 51 & 53 & 55 & 58 & 61 & 63 & 61 & 61 & 62 & 63 & 64 & 63 & 62 & 62 & 63 \\
3 & 56 & 48 & 43 & 40 & 36 & 35 & 33 & 32 & 32 & 31 & 27 & 146 & 145 & 142 & 138 & 134 & 130 & 128 & 122 & 117 & 117 & 115 & 110 & 108 \\
4 & 44 & 36 & 33 & 27 & 25 & 133 & 130 & 124 & 118 & 113 & 111 & 108 & 106 & 99 & 96 & 89 & 82 & 79 & 73 & 66 & 63 & 59 & 53 & 46 \\
5 & 52 & 56 & 63 & 71 & 79 & 89 & 96 & 106 & 113 & 122 & 127 & 139 & 143 & 152 & 161 & 171 & 178 & 190 & 17 & 23 & 35 & 47 & 54 & 64 \\
6 & 38 & 38 & 40 & 40 & 38 & 41 & 40 & 41 & 44 & 45 & 47 & 47 & 48 & 51 & 50 & 53 & 54 & 58 & 58 & 60 & 64 & 67 & 68 & 67 \\
7 & 38 & 31 & 29 & 25 & 109 & 104 & 96 & 90 & 85 & 78 & 75 & 70 & 66 & 59 & 54 & 51 & 47 & 45 & 42 & 36 & 34 & 27 & 23 & 18 \\
8 & 55 & 47 & 40 & 35 & 27 & 22 & 85 & 79 & 74 & 66 & 60 & 52 & 44 & 36 & 28 & 23 & 19 & 17 & 11 & 7 & 4 & 0 & 0 & 0 \\
\hline
\end{tabular}


TABLE 5: Records of vehicle in the optimal scheduling scheme.

\begin{tabular}{lccc}
\hline Scheduling ID & Time & Point & Loaded \\
\hline 1 & 15 & 9 & 300 \\
1 & 17.217 & 7 & 300 \\
1 & 21.717 & 7 & 210 \\
1 & 23 & 4 & 210 \\
1 & 28.5 & 4 & 100 \\
1 & 30.117 & 8 & 100 \\
1 & 33.617 & 8 & 30 \\
1 & 38.34 & 9 & 30 \\
2 & 51.84 & 9 & 300 \\
2 & 52.792 & 3 & 300 \\
2 & 58.792 & 3 & 180 \\
2 & 61.285 & 1 & 180 \\
2 & 67.285 & 1 & 300 \\
2 & 68.848 & 9 & 300 \\
3 & 83.848 & 9 & 0 \\
3 & 85.413 & 5 & 0 \\
3 & 94.613 & 5 & 184 \\
3 & 96.177 & 9 & 184 \\
\hline
\end{tabular}

\section{Conclusion}

Faced with the practical problem that the bike-sharing is difficult to borrow and return due to the lack of effective scheduling, this paper establishes a dynamic scheduling model of bike-sharing with demand prediction and scheduling optimization and proposes a model solution method based on NPGA. The model is validated by a case study. By optimizing the scheduling, the attitude of waiting, transfer, and abandoning by users when they cannot borrow a bike will be significantly reduced. The results show that rational dynamic scheduling can effectively optimize the resource allocation and improve the service level of system.

In order to simplify the model, some practical problems were not considered, including the prediction of demand, the uncertainty of scheduling time, the gender, age, travel purpose, and other personal characteristics of the users into the waiting, transfer, and abandoning probability functions. These issues will be the focus of future research.

\section{Data Availability}

The experimental data used to support the findings of this study are included within the article.

\section{Conflicts of Interest}

The authors declare no conflicts of interest regarding the publication of this paper.

\section{Acknowledgments}

This work was supported by the Fundamental Research Funds for the Central Universities (30919011290 and 30920010010) and the Natural Science Foundation of Jiangsu Province, China (BK20171426).

\section{References}

[1] Cheetah Lab, Bike-sharing Global Development Report, China, 2018, https://www.chinanews.com/cj/2018/03-07/8462117. shtml.

[2] He Liu, X. Li, and D. Chen, "An optimization model of the layout of public bike rental stations based on B+R mode," Lecture Notes in Electrical Engineering, vol. 277, pp. 13411348, 2014.

[3] S. C. Ho and W. Y. Szeto, "Solving a static repositioning problem in bike-sharing systems using iterated tabu search," Transportation Research Part E: Logistics and Transportation Review, vol. 69, pp. 180-198, 2014.

[4] M. Dell'Amico, E. Hadjicostantinou, M. Iori, and S. Novellani, "The bike sharing rebalancing problem: mathematical formulations and benchmark instances," Omega, vol. 45, pp. 7-19, 2014.

[5] J. Brinkmann, M. W. Ulmer, and D. C. Mattfeld, "Short-term strategies for stochastic inventory routing in bike sharing systems," Transportation Research Procedia, vol. 10, pp. 364373, 2015.

[6] L. Liu, L. Sun, Y. Chen, and X. Ma, "Optimizing fleet size and scheduling of feeder transit services considering the influence of bike-sharing systems," Journal of Cleaner Production, vol. 236, 2019.

[7] L. Shi, Y. Zhang, W. Rui, and X. Yang, "Study on the bikesharing inventory rebalancing and vehicle routing for bikesharing system," Transportation Research Procedia, vol. 39, pp. 624-633, 2019.

[8] T. Bulhões, A. Subramanian, G. Erdoğan, and G. Laporte, "The static bike relocation problem with multiple vehicles and visits," European Journal of Operational Research, vol. 264, 2018.

[9] J. Schuijbroek, R. C. Hampshire, and W.-J. Van Hoeve, "Inventory rebalancing and vehicle routing in bike sharing systems," European Journal of Operational Research, vol. 257, no. 3, pp. 992-1004, 2017.

[10] L. Caggiani, R. Camporeale, M. Ottomanelli, and W. Y. Szeto, "A modeling framework for the dynamic management of freefloating bike-sharing systems," Transportation Research Part C: Emerging Technologies, vol. 87, pp. 159-182, 2018.

[11] H. Guan, S. Lu, and M. Song, "Hierarchical scheduling strategy for free-floating bike-sharing," Journal of Chongqing Jiaotong University (Natural Science), vol. 39, no. 02, pp. 1-7, 2020.

[12] A. K. Ahmed, K. Imed, and L. Karim, Lower and Upper Bounds for Scheduling Multiple Balancing Vehicles in BicycleSharing Systems, Springer Berlin Heidelberg, vol. 23, no. 14, Berlin, Germany, 2019.

[13] V. Pureza, R. Morabito, and M. Reimann, "Vehicle routing with multiple deliverymen: modeling and heuristic approaches for the VRPTW," European Journal of Operational Research, vol. 218, no. 31, pp. 636-647, 2012.

[14] H. Ben Ticha, N. Absi, D. Feillet, and A. Quilliot, "Empirical analysis for the VRPTW with a multigraph representation for the road network," Computers \& Operations Research, vol. 88, pp. 103-116, 2017.

[15] M. Harzi and S. Krichen, "Variable neighborhood descent for solving the vehicle routing problem with time windows," Electronic Notes in Discrete Mathematics, vol. 58, pp. 175-182, 2017.

[16] F. Hernandez, D. Feillet, R. Giroudeau, and O. Naud, "Branch-and-price algorithms for the solution of the multitrip vehicle routing problem with time windows," European 
Journal of Operational Research, vol. 249, no. 2, pp. 551-559, 2016.

[17] D. M. Pierre and N. Zakaria, "Stochastic partially optimized cyclic shift crossover for multi-objective genetic algorithms for the vehicle routing problem with time-windows," Applied Soft Computing, vol. 52, pp. 863-876, 2017.

[18] D. Ma, J. Xiao, X. Song, X. Ma, and S. Jin, "A back-pressurebased model with fixed phase sequences for traffic signaloptimization under oversaturated networks," Institute of Electrical and Electronics Engineers Transactions on Intelligent Transportation Systems, vol. 2020, 12 pages, 2020.

[19] D. Ma, X. Song, and Li Pu, "Daily traffic flow forecasting through a contextual convolutional recurrent neural network modeling inter-and intra-day traffic patterns," Institute of Electrical and Electronics Engineers Transactions on Intelligent Transportation Systems, vol. 2020, 10 pages, 2020.

[20] K. Braekers, K. Ramaekers, and I. Van Nieuwenhuyse, "The vehicle routing problem: state of the art classification and review," Computers \& Industrial Engineering, vol. 99, pp. 300-313, 2016. 